TRANSACTIONS OF THE

AMERICAN MATHEMATICAL SOCIETY

Volume 357, Number 11, Pages 4555-4577

S 0002-9947(05)03977-2

Article electronically published on June 10, 2005

\title{
ALEKSANDROV SURFACES AND HYPERBOLICITY
}

\author{
BYUNG-GEUN OH
}

\begin{abstract}
Aleksandrov surfaces are a generalization of two-dimensional Riemannian manifolds, and it is known that every open simply-connected Aleksandrov surface is conformally equivalent either to the unit disc (hyperbolic case) or to the plane (parabolic case). We prove a criterion for hyperbolicity of Aleksandrov surfaces which have nice tilings and where negative curvature dominates. We then apply this to generalize a result of Nevanlinna and give a partial answer for his conjecture about line complexes.
\end{abstract}

\section{INTRODUCTION}

An Aleksandrov surface $S$ is an open simply-connected two-dimensional topological manifold equipped with an intrinsic metrie1 1 whose length element is locally defined by the form

$$
e^{u(z)}|d z|,
$$

where $z$ is a complex local coordinate and $u$ is a difference of two subharmonic functions such that $\exp u$ is locally integrable on rectifiable curves in the $z$-plane. A local coordinate for which (1.1) holds is called an isothermal coordinate, and isothermal coordinates define a complex analytic structure on every Aleksandrov surface. In fact by the Uniformization Theorem of Huber [15], there is an isometry

$$
h: S \rightarrow D(R):=\{z \in \mathbb{C}:|z|<R\}, \quad R \in(0, \infty],
$$

where $D(R)$ is equipped with a length element of the form (1.1). Therefore one can study complex analysis on Aleksandrov surfaces, and in this paper we give a criterion for hyperbolicity (the case $R<\infty$ in (1.2)).

To state our main theorem, we need to introduce several definitions. First, the integral curvature $\omega$ of $S$ is a signed Borel measure defined by the negative of the generalized Laplacian of $u$ (i.e., $\omega=-\Delta u$ ), where $u$ is as in (1.1). The total angle $T(v)$ at a point $v \in S$ is

$$
T(v)=2 \pi-\omega(\{v\}),
$$

and we define the angular curvature $K(\Delta)$ of a triangle $\Delta$ with vertices $v_{i}, i=1,2,3$, as the quantity

$$
K(\Delta)=2 \pi \sum_{i=1}^{3} \frac{\theta\left(v_{i}, \Delta\right)}{T\left(v_{i}\right)}-\pi,
$$

Received by the editors December 17, 2003.

2000 Mathematics Subject Classification. Primary 30F20, 30D30; Secondary 28A75, 30 D35.

${ }^{1} \mathrm{~A}$ metric is called intrinsic if the distance between two points in the space is the infimum of the lengths of curves connecting these points.

(C)2005 American Mathematical Society Reverts to public domain 28 years from publication 
where $\theta\left(v_{i}, \Delta\right)$ is the angle of $\Delta$ at $v_{i}$. A tiling $\mathcal{T}$ of $S$ is a set of triangles in $S$ which covers $S$ locally finitely and such that for different triangles $\Delta$ and $\Delta^{\prime}$ in $\mathcal{T}$, $\Delta \cap \Delta^{\prime}$ is either empty, a set of common vertices, or a set of common sides. Finally, we define a partition $\mathcal{P}$ of $\mathcal{T}$ as follows: each element $C_{\alpha} \in \mathcal{P}$, called a cluster, is a finite union of triangles in $\mathcal{T}$ with connected interior such that $C_{\alpha} \cap C_{\beta}$ contains no triangle in $\mathcal{T}$ when $\alpha \neq \beta$ and $\bigcup_{C_{\alpha} \in \mathcal{P}} C_{\alpha}=S$. Let $\#\left(C_{\alpha}\right)$ be the number of triangles in $C_{\alpha}$.

Theorem 1.5. Suppose that an Aleksandrov surface $S$ is of curvature at most $k \in \mathbb{R} 2$ If there are a tiling $\mathcal{T}$ and a partition $\mathcal{P}$ on $S$ such that for some constants $\epsilon>0$ and $M>0$,

(M1) $\#\left(C_{\alpha}\right) \leq M$ for every $C_{\alpha} \in \mathcal{P}$,

(M2) $\sum_{\Delta \subseteq C_{\alpha}} K(\Delta) \leq-\epsilon \pi$ for every $C_{\alpha} \in \mathcal{P}$,

(R1) $\theta(v, \Delta) \geq \epsilon$ for every $\Delta \in \mathcal{T}$ and every vertex $v$ of $\Delta$,

(R2) if $k>0$, then (the perimeter of $\Delta) \leq(2 \pi-\epsilon) k^{-1 / 2}$ for every $\Delta \in \mathcal{T}$,

then $S$ is hyperbolic; i.e., conformally equivalent to the unit disc.

The meaning of our main conditions (M1) and (M2) is that negative curvature dominates $S$ uniformly, and note that $S$ is allowed to have positive integral curvature in some subsets.

One cannot drop (R1), one of the regularity conditions, as Example 1.6 below shows.

Example 1.6. For every $\epsilon>0$ there exists a parabolic Aleksandrov surface of curvature at most zero which has a tiling $\mathcal{T}$ such that every triangle in $\mathcal{T}$ is isometric to a Euclidean triangle of area at most $\epsilon$ and $T(v)=4 \pi$ at every vertex $v$ of a triangle in $\mathcal{T}$.

With $M=1$, it is easy to see that the surface described in Example 1.6 satisfies all assumptions of Theorem [1.5 except (R1). This example also shows that in Proposition 1.7 of [10] the assumption about diameters of triangles cannot be replaced by an assumption about areas, while our Theorem [1.5] implies that it can be replaced by an assumption about angles. Example [1.6] will be constructed in Section 5 .

When $k>0$, we do not know whether we can drop condition (R1) or (R2). However, an Aleksandrov surface of curvature at most $k>0$ may be parabolic if it satisfies only the main conditions (M1) and (M2) of Theorem 1.5, as Example 1.2 of [8] shows: in fact, the surface constructed in $[8$, is a surface over $\mathbb{C}$ (with the induced Euclidean metric), but one can also realize it as a surface over $\overline{\mathbb{C}}$ (with the induced spherical metric, which is not complete). Then this surface becomes an Aleksandrov surface of curvature at most 1, and one can check that it satisfies conditions (M1) and (M2) only.

Let $X$ be a Riemann surface and let $f: X \rightarrow \overline{\mathbb{C}}$ be a non-constant holomorphic function. For each $p \in X$, we define $d_{f}(p)$ as the radius of the largest open spherical disc centered at $f(p)$ where the inverse of $f$ exists. Then the spherical Bloch constant $\mathcal{B}_{f}$ of $f$ is defined by the formula $\mathcal{B}_{f}:=\sup _{p \in X} d_{f}(p)$.

\footnotetext{
${ }^{2}$ This concept is defined in Section [2.1] and if $S$ is a smooth surface, then it is equivalent to the boundedness of the Gaussian curvature from above by $k$.
} 
Theorem 1.7 (M. Bonk and A. Eremenko [8], Proposition 8.4). Let $X$ be a Riemann surface and let $f: X \rightarrow \overline{\mathbb{C}}$ be a non-constant holomorphic function without asymptotic values such that $\mathcal{B}_{f} \leq \pi / 2-\epsilon$ for some $\epsilon>0$. Then there exists a tiling $\mathcal{T}$ of $X$ with respect to the pull-back spherical metric such that the image $f(\Delta)$ of every triangle $\Delta \in \mathcal{T}$ is contained in a closed spherical disc of radius $\mathcal{B}_{f}$ and the set of all the vertices of the triangles in $\mathcal{T}$ is equal to the set of critical points of $f$.

Theorem 1.7 gives a sufficient condition for the existence of a tiling, which is apriori assumed in our Theorem [1.5. Also note that if $S$ is a Riemann surface spread over $\overline{\mathbb{C}}$ and ramified only over finitely many points, then a tiling always exists with respect to the pull-back spherical metric (cf. Section [6).

M. Bonk and A. Eremenko conjectured in [10] (cf. [7]) the following statement:

Suppose that $S$ is an open simply-connected complete Aleksandrov surface with a tiling $\mathcal{T}$ such that for some $q \in(1,3]$ and $\epsilon>0$, the following two conditions are satisfied:

(1) every triangle $\Delta \in \mathcal{T}$ is isometric to a spherical triangle with circumscribed radius at most

$$
\mathcal{R}_{q, \epsilon}:=\arctan \sqrt{\frac{-\cos (\pi q / 2)}{\cos ^{3}(\pi q / 6)}}-\epsilon ;
$$

(2) the total angle at each vertex is at least $2 \pi q$. Then $S$ is of hyperbolic type.

The expression $\mathcal{R}_{q, \epsilon}$ in (1.8) with $\epsilon=0$ is the circumscribed radius of a spherical equilateral triangle with angles $\pi q / 3$.

M. Bonk and A. Eremenko proved this conjecture in [10] for the cases $q=2,3$ and for the limiting case $q \rightarrow 1$ with an appropriate interpretation. This conjecture still remains open for general $q \in(1,3]$, but as one of the applications of Theorem 1.5 we have the following corollary:

Corollary 1.9. Suppose $S$ is an Aleksandrov surface as described in the above conjecture with the additional assumption (R1) of Theorem [1.5. Then $S$ is of hyperbolic type.

Proof. It suffices to check the four conditions in Theorem 1.5 because every triangle in $\mathcal{T}$ is spherical.

First, we observe that the area of every spherical triangle with circumscribed radius at most $\mathcal{R}_{q, \epsilon}$ is less than or equal to $\pi(q-1)-\eta$, where $\eta>0$ is a constant depending only on $\epsilon$. Therefore every triangle $\Delta \in \mathcal{T}$ has angular curvature

$$
K(\Delta) \leq 2 \pi \frac{\pi q-\eta}{2 \pi q}-\pi=-\frac{\eta}{q}
$$

so conditions (M1) and (M2) of Theorem 1.5 are satisfied with $M=1$. Note that we already assumed condition (R1) in this corollary. Condition (R2) is trivially satisfied because the circumscribed radius of every triangle $\Delta \in \mathcal{T}$ is at most $\mathcal{R}_{q, \epsilon} \leq \pi / 2-\epsilon$.

More applications will be given in Secion [6.

\section{Preliminaries}

2.1. Aleksandrov surfaces of curvature at most $k$. Let $S$ be an Aleksandrov surface with an intrinsic metric $\rho$. A curve joining points $x, y \in S$ is called a shortest curve if its length is equal to the distance $d_{\rho}(x, y)$ between $x$ and $y$, and is denoted 
by $[x, y]$. (This notation is ambiguous because we do not require the uniqueness of shortest curves.)

A geodesic triangle $\Delta=\Delta\left(v_{1} v_{2} v_{3}\right)$ in $S$ is a closed set which is homeomorphic to the closed unit disc and whose boundary $\partial \Delta$ consists of three shortest curves $\left[v_{1}, v_{2}\right],\left[v_{2}, v_{3}\right],\left[v_{3}, v_{1}\right]$, called the sides of $\Delta$. Each point $v_{i}$ is called a vertex of $\Delta$, and the perimeter of $\Delta$ is the sum $d_{\rho}\left(v_{1}, v_{2}\right)+d_{\rho}\left(v_{2}, v_{3}\right)+d_{\rho}\left(v_{3}, v_{1}\right)$. A geodesic triangle $\Delta$ is called a simple (geodesic) triangle if for any two points $x, y \in \Delta$ there exists a shortest curve $[x, y] \subseteq \Delta$.

Now suppose $L$ and $M$ are two curves in $S$ that have a common starting point $o$. On $L$ and $M$ we choose arbitrary points $x$ and $y$, respectively, and let

$$
X=d_{\rho}(o, x), \quad Y=d_{\rho}(o, y), \quad Z=d_{\rho}(x, y) .
$$

We then construct a triangle in the Euclidean plane with side-lengths $X, Y$ and $Z$, and let $\gamma_{L M}(X, Y)$ be the angle opposite to the side of length $Z$ in this Euclidean triangle. Then the upper angle between $L$ and $M$ is

$$
\overline{\angle(L, M)}=\limsup _{X, Y \rightarrow 0} \gamma_{L M}(X, Y) \in[0, \pi] .
$$

Using this definition of upper angles, one can define (upper) angles of a geodesic triangle at each of its vertices. We will mostly use the terminology "angles" instead of "upper angles" when they are associated to "small" simple triangles, because for any two "small" shortest curves the angle between them exists; i.e., the limit of $\gamma_{L M}(X, Y)$ in (2.1) exists as $X$ and $Y$ go to zero ([6], pp. 176-177, 182, 187).

Now fix a number $k \in \mathbb{R}$. We denote by $S(k)$ the Euclidean plane when $k=0$, the hyperbolic plane of curvature $k$ when $k<0$, and a two-dimensional open hemisphere of radius $k^{-1 / 2}$ when $k>0$. Then for a given geodesic triangle $\Delta$ in $S$, we associate a new triangle $\Delta_{k}$ on $S(k)$ which has the same side-lengths as $\Delta$. (When $k \leq 0$ the triangle $\Delta_{k}$ exists because of the triangle inequality, and when $k>0$ it is necessary to require that the perimeter of $\Delta$ is less than $2 \pi k^{-1 / 2}$. When $k>0$ it will be assumed throughout that this requirement is satisfied.) If $\Delta$ has upper angles $\bar{\alpha}, \bar{\beta}, \bar{\gamma}$ and the corresponding angles of $\Delta_{k}$ are $\alpha_{k}, \beta_{k}, \gamma_{k}$, then the $k$-excess of $\Delta$ is the quantity

$$
\delta_{k}(\Delta)=(\bar{\alpha}+\bar{\beta}+\bar{\gamma})-\left(\alpha_{k}+\beta_{k}+\gamma_{k}\right) .
$$

Definition 2.2. A domain $R_{k}$ is an intrinsic metric space satisfying the following properties:

(a) any two points in $R_{k}$ can be joined by a shortest curve;

(b) each geodesic triangle in $R_{k}$ has non-positive $k$-excess;

(c) if $k>0$, the perimeter of each geodesic triangle in $R_{k}$ is less than $2 \pi k^{-1 / 2}$.

We say that an Aleksandrov surface $S$ is of curvature at most $k$ if every point in $S$ has a neighborhood which is a domain $R_{k}$, and denote by $\mathfrak{S}(k)$ the collection of Aleksandrov surfaces of curvature at most $k$. Note that this notion is equivalent to the boundedness of the Gaussian curvature from above (by $k$ ) when $S$ is a Riemannian surface, and also note that if an Aleksandrov surface has a point $v$ such that $\omega(\{p\})>0$, or equivalently $T(p)<2 \pi$, then it does not belong to $\mathfrak{S}(k)$ for any $k<\infty$. 
2.2. Generalized simple geodesic triangles. Let $\bar{S}$ be the compactification of $S \in \mathfrak{S}(k)$ with respect to the given metric $\rho$. A point $x \in \bar{S}$ is called a point at infinity if $x \notin S$, and it is called a point in $S$ or a finite point otherwise. A rectifiable curve $\gamma: I \rightarrow S$, where $I$ is one of the intervals $(0,1),[0,1),(0,1]$ or $[0,1]$, is called a locally shortest curve if its extension $\bar{\gamma}: \bar{I} \rightarrow \bar{S}$ defines a shortest curve in $\bar{S}$. A locally shortest curve with endpoints $x, y \in \bar{S}$ will be denoted by $[x, y]$ as in the case of shortest curves.

Definition 2.3. A generalized simple geodesic triangle, or just a triangle for brevity, on an Aleksandrov surface $S \in \mathfrak{S}(k)$ is a closed (not necessarily compact) subset $\Delta$ of $S$ such that its interior is homeomorphic to the open unit disc and it satisfies the following properties:

(a) the boundary of $\Delta$ consists of three locally shortest curves, called sides of $\Delta$

(b) each pair of sides of $\Delta$ has one and only one common endpoint in $\bar{S}$, called a vertex of $\Delta$

(c) the $\bar{S}$-closure $\bar{\Delta}$ of $\Delta$ is a domain $R_{k}$.

Roughly speaking, a triangle is a simple triangle (as defined in Section 2.11) with some vertices deleted, and we require that it is also small to be a domain $R_{k}$. Also note that condition (c) above makes it possible to define an angle of $\Delta$ at a vertex at infinity by the formula (2.1).

Throughout this paper triangles in $S$ always mean the sets described in Definition 2.3, while triangles in the "model domain" $S(k)$ are the usual compact triangles in the Euclidean plane, hemispheres or hyperbolic planes.

2.3. Total angle and angular curvature. In this subsection we discuss the meaning of total angle and angular curvature. To do this, suppose $S \in \mathfrak{S}(k)$ with a tiling $\mathcal{T}$. For a vertex $v \in \bar{S}$ of a triangle $\Delta \in \mathcal{T}$, we denote by $\theta(v, \Delta)$ the angle of $\Delta$ at $v$ as before, and define the total angle $T(v)$ of $v$ to be

$$
T(v):=\sum_{\Delta \in \mathcal{T}} \theta(v, \Delta)
$$

with the convention that $\theta(v, \Delta)=0$ if $v$ is not a vertex of $\Delta$. By the Gauss-Bonnet formula ([4, p. 214) one can check that this definition of total angle coincides with (1.3) for a finite vertex $v$. Also note that (2.4) is valid for a vertex $v$ at infinity by defining $T(v)=\infty$. In fact if we assume condition (R1) of Theorem 1.5, then because $S$ is simply connected the case $T(v)<\infty$ cannot happen for a vertex $v$ at infinity, unless $\mathcal{T}$ contains only finitely many triangles. But then $S \cup\{v\}$ becomes compact, hence $S$ is parabolic. In addition, one may check that $\sum_{\Delta \in \mathcal{T}} K(\Delta)=4 \pi$ in this case and condition (M2) of Theorem 1.5 is never satisfied. So without loss of generality, we will always assume that $T(v)=\infty$ for a vertex $v$ at infinity.

The reason why we consider the angular curvature of a triangle is the following. For a given triangle $\Delta \in \mathcal{T}$, we distribute to $\Delta$ the integral curvature concentrated at its vertices, weighted by the angle at each vertex. Thus if $\Delta$ has vertices $v_{i}$ and sides $L_{i}, i=1,2,3$, the integral curvature of the closed triangle $\Delta$ would be (with the interpretation $\infty / \infty=1$ and $a / \infty=0$ for $a<\infty$ )

$$
\sum_{i=1}^{3} \frac{2 \pi-T\left(v_{i}\right)}{T\left(v_{i}\right)} \theta\left(v_{i}, \Delta\right)+\omega\left(\Delta^{\circ}\right)+\sum_{i=1}^{3} \tau\left(L_{i}\right),
$$


where $\tau\left(L_{i}\right)$ is the so-called left turn (cf. 4] or [18) of the shortest curve $L_{i}$. (The reason we have the left turn of sides of $\Delta$ in (2.5) is the same as before: we want to compute the integral curvature of a closed triangle.) On the other hand, the Gauss-Bonnet formula applied to a triangle $\Delta$ implies that

$$
\sum_{i=1}^{3} \theta\left(v_{i}, \Delta\right)-\pi-\sum_{i=1}^{3} \tau\left(L_{i}\right)=\omega\left(\Delta^{\circ}\right) .
$$

Now one can easily see that the quantity in (2.5) is same as the angular curvature $K(\Delta)$ defined in (1.4).

2.4. Überlagerungsfläche. Let $X$ and $Y$ be two-dimensional topological manifolds and let $\varphi: X \rightarrow Y$ be a topologically holomorphis 3 map. Then by a theorem of Stoilov [19], for every $x \in X$ there are a positive integer $m$, called the local degree of $\varphi$ at $x$, and complex local coordinates $z$ and $w$ in neighborhoods of $x$ and $\varphi(x)$ such that $w(\varphi(x))=z(x)^{m}$. Therefore if $Y$ is a Riemann surface, there exists a unique conformal structure on $X$ which makes $\varphi$ holomorphic, and the pair $(X, \varphi)$ is called a Riemann surface spread over $Y$ (Überlagerungsfläche in German). Two such pairs $\left(X_{1}, \varphi_{1}\right)$ and $\left(X_{2}, \varphi_{2}\right)$ are called equivalent if there is a homeomorphism $h: X_{1} \rightarrow X_{2}$ such that $\varphi_{1}=\varphi_{2} \circ h$, and strictly speaking, a Riemann surface spread over $Y$ is an equivalence class of such pairs.

Now suppose $(X, \varphi)$ is an open simply-connected Riemann surface spread over $Y=\mathbb{C}, \overline{\mathbb{C}}$ or $\mathbb{D}$. Then the surface $X$ equipped with the pull-back metric, i.e., the metric whose length element is of the form

$$
\frac{2\left|\varphi^{\prime}(z)\right||d z|}{1+\chi|\varphi(z)|^{2}}
$$

with $\chi=0,1$ or -1 depending on whether $Y$ is $\mathbb{C}, \overline{\mathbb{C}}$ or $\mathbb{D}$, respectively, is a special type of an Aleksandrov surface, called a surface with polyhedral metric [18. In this connection the study of Aleksandrov surfaces naturally arises in the field of function theory. Also note that the Riemann surface $X$ equipped with the metric defined by (2.7) is in $\mathfrak{S}(\chi)$.

2.5. Notation. We always use the letter $\rho$ to denote the intrinsic metric of a given Aleksandrov surface $S$, or the length element of this metric. The area of a region in $S$ will be denoted by $|\cdot|_{\rho}$, the length of a curve in $S$ by $\ell_{\rho}(\cdot)$, and the distance between two points $x$ and $y$ in $S$ by $d_{\rho}(x, y)$. When we consider the metric $\lambda$, which will be constructed in Section 4 , the subscript $\rho$ will be modified to $\lambda$.

\section{Three Lemmas}

We first state several theorems of A. D. Aleksandrov, which will be used in the proof of Theorem 1.5

Theorem 3.1 (Aleksandrov [3]). The upper angles $\bar{\alpha}, \bar{\beta}, \bar{\gamma}$ of an arbitrary triangle $\Delta$ in a domain $R_{k}$ are not greater than the corresponding angles $\alpha_{k}, \beta_{k}, \gamma_{k}$ of the associated triangle $\Delta_{k}$ on $S(k)$.

Note that this theorem says that each of the angles of $\Delta$ is less than or equal to the corresponding angle of $\Delta_{k}$, while the second condition of Definition 2.2 says that only the sum of angles of $\Delta$ is at most that of $\Delta_{k}$.

\footnotetext{
${ }^{3}$ Continuous, open and discrete.
} 
Theorem 3.2 (Aleksandrov [3]). Any two points of $R_{k}$ are joined by a unique shortest curve.

Theorem 3.3 (Aleksandrov [2]). Let $P$ be a polygon in $S \in \mathfrak{S}(k), k>0$. If the perimeter of $P$ is less than $2 \pi k^{-1 / 2}$ and every two points in $P$ can be joined by a unique shortest curve in $P$, then the area of $P$ does not exceed that of the polygon $P_{0}$ which has sides of the same length and is inscribed in a circle on a hemisphere of curvature $k$.

Note that Theorems 3.2 and 3.3 in particular imply that any triangle $\Delta \subseteq S \in$ $\mathfrak{S}(k), k>0$, has area at most $2 \pi k^{-1}$.

In the following two sections, $S$ will represent an Aleksandrov surface of curvature at most $k$ satisfying all properties of Theorem 1.5 All notations in Theorem 1.5 (such as $\mathcal{T}, \mathcal{P}$ ) and in Section 2.5 will be used without further remarks.

Lemma 3.4. Let $\Delta$ be a triangle in $\mathcal{T}$ and let $\Gamma$ be a Jordan arc contained in $\Delta$ with endpoints on $\partial \Delta$. Among the two subarcs of $\partial \Delta$ (or $\partial \bar{\Delta}$ if $\Delta$ has some vertices at infinity) divided by $\Gamma$, we take the subarc $\Lambda$ which contains the smaller number of vertices. (If both subarcs contain the same number of vertices, we choose either.) Then there exists a constant $C=C(\epsilon)$ such that

$$
\ell_{\rho}(\Lambda) \leq C \ell_{\rho}(\Gamma) .
$$

Proof. Because every side of $\Delta$ is a locally shortest curve, there is nothing to prove if the endpoints of $\Gamma, x$ and $y$, are on a same side of $\Delta$. So we assume that $x$ and $y$ are on different sides of $\Delta$. In this case, $\Lambda$ must contain one and only one vertex of $\Delta$, denoted by $z$, in its interior arc.

Let $\widetilde{\Delta}$ be the triangle contained in $\Delta$ with vertices $x, y$ and $z$. We denote by $a$, $b$ and $c$ the length of sides of $\widetilde{\Delta}$ which are opposite to $x, y$ and $z$, respectively, and by $\alpha, \beta$ and $\gamma$ the angles of $\widetilde{\Delta}$ at $x, y$ and $z$, respectively. Now if we show that

$$
\max \{a, b\} \leq C(\epsilon) \cdot c
$$

for some constant $C(\epsilon)>0$, then we have proved the lemma since

$$
\begin{aligned}
\ell_{\rho}(\Lambda) & =d_{\rho}(x, z)+d_{\rho}(y, z)=a+b \leq 2 C(\epsilon) \cdot c \\
& =2 C(\epsilon) d_{\rho}(x, y) \leq 2 C(\epsilon) \ell_{\rho}(\Gamma) .
\end{aligned}
$$

To show (3.5) we first note that it can be assumed that $k \in\{0,1\}$. In fact, we may take $k=0$ if $k \leq 0$ because $S \in \mathfrak{S}(k) \subseteq \mathfrak{S}(0)$. If $k>0$, then a surface $S \in \mathfrak{S}(k)$ with the given metric $\rho$ can be realized as a surface in $\mathfrak{S}(1)$ with the metric $\sqrt{k} \rho$, so in this case $k$ can be assumed to be 1 .

We next construct a new triangle $\widetilde{\Delta}_{k}$ on $S(k)$ whose side lengths are those of $\widetilde{\Delta}$. Theorem 3.1 then yields that the corresponding angles $\alpha_{k}, \beta_{k}$ and $\gamma_{k}$ are greater than or equal to $\alpha, \beta$ and $\gamma$, respectively. In particular, $\gamma_{k} \geq \gamma \geq \epsilon$, since $\gamma$ is an angle of $\Delta \in \mathcal{T}$ and $\mathcal{T}$ satisfies condition (R1) of Theorem 1.5. Furthermore, one may assume that $\gamma_{k} \leq \pi / 2$ by dividing $\widetilde{\Delta}_{k}$ into two triangles with the bisector of $\gamma_{k}$ if necessary, because our goal here is to show (3.5) in the triangle $\widetilde{\Delta}_{k}$. Thus

$$
\sin \gamma_{k} \geq \sin \epsilon .
$$

Now if $k=0$, the Law of Sines and (3.6) imply

$$
b=\frac{\sin \beta_{k}}{\sin \gamma_{k}} c \leq \frac{1}{\sin \gamma_{k}} c \leq \frac{1}{\sin \epsilon} c,
$$


and similarly $a \leq(\sin \epsilon)^{-1} c$, as desired. If $k=1$, then since the perimeter of $\Delta$ is bounded by $2 \pi-\epsilon$ by assumption (R2) with $k=1$, we have $0 \leq b \leq \pi-\epsilon / 2$, or

$$
b \leq \frac{\pi-\epsilon / 2}{\sin (\pi-\epsilon / 2)} \sin b \leq \frac{\pi}{\sin (\epsilon / 2)} \sin b .
$$

On the other hand, by the Spherical Law of Sines (cf. [12, p. 151)

$$
\frac{\sin a}{\sin \alpha}=\frac{\sin b}{\sin \beta}=\frac{\sin c}{\sin \gamma}
$$

and (3.6) we have

$$
\sin b=\frac{\sin \beta_{k}}{\sin \gamma_{k}} \sin c \leq \frac{1}{\sin \epsilon} \sin c \leq \frac{c}{\sin \epsilon} .
$$

Therefore, combining (3.7) and (3.8), $b \leq \pi(\sin (\epsilon / 2) \sin (\epsilon))^{-1} c$, and similarly $a \leq$ $\pi(\sin (\epsilon / 2) \sin (\epsilon))^{-1} c$. This completes the proof.

The proof of this lemma shows that $\max \{a, b\} \leq C(\epsilon) \cdot c$ if $\gamma \geq \epsilon$ and the perimeter of $\widetilde{\Delta}$ is at most $(2 \pi-\epsilon) k^{-1 / 2}$ for $k>0$. Then since $\min \{\alpha, \beta, \gamma\} \geq \epsilon$ when $\widetilde{\Delta}=\Delta$, we have the following corollary.

Corollary 3.9. For every $\Delta \in \mathcal{T}$ there exists a constant $C=C(\epsilon)$ such that $M(\Delta) \leq C m(\Delta)$, where

$$
\begin{aligned}
M(\Delta) & :=\max \{\text { side-lengths of } \Delta\}, \\
m(\Delta) & :=\min \{\text { side-lengths of } \Delta\} .
\end{aligned}
$$

We next have an isoperimetric inequality for small domains.

Lemma 3.11. Suppose $D$ is an open set which is contained in a single triangle $\Delta \in \mathcal{T}$. Then

$$
|D|_{\rho} \leq \frac{1}{2 \pi} \ell_{\rho}(\partial D)^{2} .
$$

Proof. Without loss of generality, we may assume that all vertices of $\Delta$ are finite, because otherwise the argument below holds with the $\bar{S}$-closure $\bar{\Delta}$ in place of $\Delta$.

Now if all vertices of $\Delta$ are finite, by considering each component of $D$ separately and by adding to $D$ all complementary components of $D$ which are compactly contained in $\Delta$, we may assume that $D$ is simply connected. Since $S \in \mathfrak{S}(k)$, we have (11], p. 12)

$$
\ell_{\rho}(\partial D)^{2}-4 \pi|D|_{\rho}+k|D|_{\rho}^{2} \geq 0 .
$$

This directly implies (3.12) when $k \leq 0$.

If $k>0$, then by the statement following Theorem 3.3 we have

$$
|D|_{\rho} \leq|\Delta|_{\rho} \leq \frac{2 \pi}{k} .
$$

This inequality together with (3.13) implies

$$
2 \pi|D|_{\rho} \leq\left(4 \pi-k|D|_{\rho}\right)|D|_{\rho} \leq \ell_{\rho}(\partial D)^{2},
$$

as desired.

The following lemma is the only place where conditions (M1) and (M2) of Theorem 1.5 are used, even though these conditions are our main assumptions. 
Lemma 3.14. Suppose that $S$ is an Aleksandrov surface with a tiling $\mathcal{T}$ and a partition $\mathcal{P}$ such that conditions (M1) and (M2) in Theorem 1.5 hold. Let $\mathcal{T}^{\prime}$ be a finite subset of $\mathcal{T}$ and let $D:=\left(\bigcup_{\Delta \in \mathcal{T}}, \Delta\right)^{\circ}$. Define

$$
\begin{aligned}
e_{0} & :=\text { the number of edges on } \partial D, \\
f & :=\text { the number of triangles in } \bar{D} .
\end{aligned}
$$

Then

$$
f \leq \frac{6 M^{2}}{\epsilon} e_{0},
$$

where $M$ and $\epsilon$ are the constants in Theorem 1.5

Proof. We first assume that $D$ consists of clusters in $\mathcal{P}$; i.e., $D$ is of the form

$$
D=\left(\bigcup_{i=1}^{N} C_{\alpha_{i}}\right)^{\circ},
$$

for some $C_{\alpha_{i}} \in \mathcal{P}, i=1, \ldots, N$. Then because the interior of each cluster in $\mathcal{P}$ is connected, all components of $D$ and all the components of $(S \backslash \bar{D})$ which consist of finitely many triangles are also unions of clusters. That is, they are also expressed in the same way as formula (3.15). Therefore to prove the lemma in this case, we may assume that $D$ is simply connected by adding to $D$ all the complementary components of $D$ consisting of finitely many triangles, and considering each component of $D$ separately.

Let $V^{\prime}$ be the set of vertices lying in the interior of $D$ and let $v^{\prime}:=\left|V^{\prime}\right|$. (Note that $V^{\prime}$ does not contain any vertices at infinity because $f$ is finite.) Then $e_{0}+v^{\prime}$ is the number of all vertices in the $\bar{S}$-closure $\bar{D}$, since for simply-connected $D$ the number of edges on $\partial D$ is same as that of vertices on $\partial \bar{D}$. Let $e$ be the number of edges of triangles in $\bar{D}$. Euler's formula then gives

$$
e_{0}+v^{\prime}+f=e+1 \text {. }
$$

On the other hand, each triangle has three edges and each edge corresponds to two different triangles except those on $\partial D$, each of which corresponds to only one triangle. Thus

$$
3 f+e_{0}=2 e .
$$

Combining this with (3.16), we get

$$
e_{0}=f-2 v^{\prime}+2 \text {. }
$$

Now since $V^{\prime}$ contains no vertices at infinity, we have $T(v)<\infty$ for all $v \in V^{\prime}$. Therefore we have

$$
\begin{aligned}
2 \pi v^{\prime}-\pi f & =2 \pi\left(\sum_{v \in V^{\prime}} \frac{T(v)}{T(v)}\right)-\pi f=2 \pi \sum_{v \in V^{\prime}} \frac{1}{T(v)}\left(\sum_{\Delta \in \mathcal{T}^{\prime}} \theta(v, \Delta)\right)-\pi f \\
& \leq \sum_{\Delta \in \mathcal{T}^{\prime}}\left(2 \pi \sum_{i=1}^{3} \frac{\theta\left(v_{i}, \Delta\right)}{T\left(v_{i}\right)}\right)-\pi f=\sum_{\Delta \in \mathcal{T}^{\prime}} K(\Delta) \leq-\epsilon \pi N
\end{aligned}
$$

or

$$
2 v^{\prime} \leq f-\epsilon N
$$


This inequality and (3.17) imply

$$
e_{0} \geq f-(f-\epsilon N)=\epsilon N \geq \frac{\epsilon}{M} f,
$$

because $f$ is less than or equal to $M N$.

If $D$ is not a union of clusters, let $\left\{C_{1}, \ldots, C_{s}\right\}$ be the largest subset of $\mathcal{P}$ such that $C_{i} \cap \partial D$ contains an edge for all $i=1, \ldots, s$. Now let $\mathfrak{D}:=\left(D \cup \bigcup_{i=1}^{s} C_{i}\right)^{\circ}$, and note that $\mathfrak{D}$ has the form 3.15) because the interior of every cluster in $\mathcal{P}$ is connected. Define

$$
\begin{aligned}
\tilde{e_{0}} & :=\text { the number of edges on } \partial \mathfrak{D}, \\
\tilde{f} & :=\text { the number of triangles in } \overline{\mathfrak{D}} .
\end{aligned}
$$

Because $\partial D$ has $e_{0}$ edges, each of which corresponds to at most two clusters, $s$ must be less than or equal to $2 e_{0}$. On the other hand, every edge on $\partial \mathfrak{D}$ must be an edge in $C_{i}$, for some $i=1, \ldots, s$. Furthermore each $C_{i}$ has at most $3 M$ edges since it contains at most $M$ triangles. Therefore,

$$
\tilde{e_{0}} \leq 3 M s \leq 6 M e_{0} .
$$

Since $f \leq \tilde{f} \leq(M / \epsilon) \tilde{e_{0}}$ by (3.18), we get

$$
f \leq \frac{6 M^{2}}{\epsilon} e_{0}
$$

as desired.

\section{Proof of Theorem 1.5}

For any $\Delta \in \mathcal{T}$, let $e$ be a side of $\Delta$. We define

$$
\lambda_{0}(z):=\frac{1}{\ell_{\rho}(e)} \quad \text { for all } z \in e \backslash\{\text { endpoints of } e\} .
$$

We define $\lambda_{0}$ for the other sides of $\Delta$ in the same way and extend $\lambda_{0}$ to the interior of $\Delta$ as the bounded solution of the Dirichlet problem

$$
\begin{aligned}
\Delta u=0 & \text { in } \Delta^{\circ}, \\
u(z)=\lambda_{0}(z) & \text { for } z \in \partial \Delta \backslash\{\text { vertices of } \Delta\} .
\end{aligned}
$$

We either leave $\lambda_{0}$ undefined on vertices or define it to be zero. Finally we define the metric $\lambda$ as the metric whose length element is $\lambda_{0} \rho$. This length element is also denoted by $\lambda$.

Note that for any triangle $\Delta \in \mathcal{T}$ and any side $e$ of $\Delta$,

$$
\ell_{\lambda}(e)=1
$$

by the definition of $\lambda$. Furthermore by Phragmén-Lindelöf's maximum principle ([1], p. 38),

$$
\frac{1}{M(\Delta)} \rho(z) \leq \lambda(z) \leq \frac{1}{m(\Delta)} \rho(z) \quad \text { for a.e. } z \in \Delta,
$$

where $M(\Delta)$ and $m(\Delta)$ are defined in (3.10). It follows that for any Borel set $D \subseteq \Delta$ and any rectifiable curve $\Gamma \subseteq \Delta$,

$$
\begin{aligned}
& \frac{1}{M(\Delta)^{2}}|D|_{\rho} \leq|D|_{\lambda} \leq \frac{1}{m(\Delta)^{2}}|D|_{\rho}, \\
& \frac{1}{M(\Delta)} \ell_{\rho}(\Gamma) \leq \ell_{\lambda}(\Gamma) \leq \frac{1}{m(\Delta)} \ell_{\rho}(\Gamma) .
\end{aligned}
$$


Lemma 4.4. Suppose $D$ is an open set in $S$ which is contained in a triangle $\Delta \in \mathcal{T}$. Then $|D|_{\lambda} \leq C \ell_{\lambda}(\partial D)$ for some $C=C(\epsilon)$.

Proof. First we assume that $\ell_{\lambda}(\partial D) \geq 1$. Then by Lemma 3.11, Corollary 3.9 and (4.1) - (4.3),

$$
\begin{aligned}
|D|_{\lambda} & \leq|\Delta|_{\lambda} \leq \frac{1}{m(\Delta)^{2}}|\Delta|_{\rho} \leq \frac{1}{m(\Delta)^{2}} \frac{1}{2 \pi} \ell_{\rho}(\partial \Delta)^{2} \\
& \leq \frac{M(\Delta)^{2}}{2 \pi m(\Delta)^{2}} \ell_{\lambda}(\partial \Delta)^{2} \leq \frac{9 C^{2}}{2 \pi} \leq \frac{9 C^{2}}{2 \pi} \ell_{\lambda}(\partial D),
\end{aligned}
$$

as desired. If $\ell_{\lambda}(\partial D)<1$, a similar calculation shows

$$
\begin{aligned}
|D|_{\lambda} & \leq \frac{1}{m(\Delta)^{2}}|D|_{\rho} \leq \frac{1}{m(\Delta)^{2}} \frac{1}{2 \pi} \ell_{\rho}(\partial D)^{2} \\
& \leq \frac{M(\Delta)^{2}}{2 \pi m(\Delta)^{2}} \ell_{\lambda}(\partial D)^{2} \leq \frac{C^{2}}{2 \pi} \ell_{\lambda}(\partial D),
\end{aligned}
$$

and the lemma follows.

In (4.5), we showed that for any $\Delta \in \mathcal{T},|\Delta|_{\lambda} \leq\left(9 C^{2}\right) /(2 \pi)$. Therefore by (4.1) and Lemma 3.14, we deduce the following corollary.

Corollary 4.6. As in Lemma 3.14 let $D$ be an open set consisting of a finite number of triangles in $\mathcal{T}$. Then there exists a constant $C=C(\epsilon, M)$ such that

$$
|D|_{\lambda} \leq C \ell_{\lambda}(\partial D) \text {. }
$$

Now suppose that a Jordan region $D$ in $S$ is given and $\Delta$ is a triangle in $\mathcal{T}$ such that $\partial D \cap \Delta^{\circ} \neq \emptyset$ and $D \nsubseteq \Delta$. Then $\Gamma:=\Delta^{\circ} \cap \partial D$ is a countable union of Jordan arcs in $\Delta$ with endpoints on $\partial \Delta$; i.e., $\Gamma=\bigcup_{j} \Gamma_{j}$ where $\Gamma_{i} \cap \Gamma_{j}=\emptyset$ if $i \neq j$, and each $\Gamma_{j}$ is a Jordan arc in $\Delta$ with endpoints on $\partial \Delta$.

For each $\Gamma_{j}$, let $\Lambda_{j}$ be one of the two closed subarcs of $\partial \bar{\Delta}$ which contains the smaller number of vertices, as in Lemma 3.4, and let $U_{j}$ be the subregion of $\Delta$ enclosed by $\Gamma_{j}$ and $\Lambda_{j}$. Note that in our notation, $D$ and each $U_{j}$ are open regions, each $\Gamma_{j}$ is an open arc, while each $\Lambda_{j}$ and $\Delta$ are closed sets. Finally let us define $\alpha:=\partial \Delta \cap D$ and $\beta:=\partial \Delta \backslash \bar{D}$.

Lemma 4.7. Either $\alpha \subseteq \bigcup_{j} \Lambda_{j}$ or $\beta \subseteq \bigcup_{j} \Lambda_{j}$. If $\beta \nsubseteq \bigcup_{j} \Lambda_{j}$, then $D \cap \Delta \subseteq \bigcup_{j} \bar{U}_{j}$.

Proof. Suppose that the first statement is not true. Then there exist $x$ and $y$ such that $x \in \alpha \backslash \bigcup_{j} \Lambda_{j}$ and $y \in \beta \backslash \bigcup_{j} \Lambda_{j}$. Since $x \in D$ and $y \in S \backslash \bar{D}$, we can find a Jordan $\operatorname{arc} \Gamma_{j}$ which separates $x$ and $y$ in $\Delta$. Then either $x \in \Lambda_{j}$ or $y \in \Lambda_{j}$ by the definition of $\Lambda_{j}$, but this contradicts our assumption.

Now suppose that $\beta \nsubseteq \bigcup_{j} \Lambda_{j}$. Then there exists $y \in \beta \backslash \bigcup_{j} \Lambda_{j}$. So for any $x \in D \cap \Delta$, either $x \in \bar{U}_{j}$ or $y \in \bar{U}_{j}$ for some $j$, by the same argument as above. But $y \notin \Lambda_{j}=\bar{U}_{j} \cap \partial \Delta$, which implies that $x \in \bar{U}_{j} \subseteq \bigcup_{j} \bar{U}_{j}$. So $D \cap \Delta \subseteq \bigcup_{j} \bar{U}_{j}$, as desired.

If $\beta \nsubseteq \bigcup_{j} \Lambda_{j}$, we have $\partial(D \backslash \Delta) \subseteq \partial D \cup \alpha$. In fact, if $x \in \partial(D \backslash \Delta) \backslash \partial D$, then $x \in$ $(\overline{D \backslash \Delta}) \subseteq \bar{D} \cap(\overline{S \backslash \Delta}), x \notin D \backslash \Delta$ and $x \notin \bar{D} \backslash D$. In particular, $x \in \bar{D}, x \notin \bar{D} \backslash D$ and $x \notin D \backslash \Delta$. So $x \in D \cap \Delta$. But since $x \in(\overline{S \backslash \Delta})$, we conclude that $x \in D \cap \partial \Delta=\alpha$. 
In this case, we claim that for some constant $C=C(\epsilon)$,

$$
\begin{aligned}
& \ell_{\lambda}(\partial(D \backslash \Delta)) \leq \ell_{\lambda}(\partial D)+\ell_{\lambda}(\alpha) \leq \ell_{\lambda}(\partial D)+C \ell_{\lambda}(\Gamma), \\
& |D \backslash \Delta|_{\lambda} \geq|D|_{\lambda}-|D \cap \Delta|_{\lambda} \geq|D|_{\lambda}-C \ell_{\lambda}(\Gamma) .
\end{aligned}
$$

In fact, since $\beta \nsubseteq \bigcup_{j} \Lambda_{j}$ we have $\alpha \subseteq \bigcup_{j} \Lambda_{j}$ by Lemma4.7 Therefore by (4.3) and Lemma 3.4 we have

$$
\begin{aligned}
\ell_{\lambda}(\alpha) & \leq \sum_{j} \ell_{\lambda}\left(\Lambda_{j}\right) \leq \sum_{j} \frac{1}{m(\Delta)} \ell_{\rho}\left(\Lambda_{j}\right) \leq C \sum_{j} \frac{1}{m(\Delta)} \ell_{\rho}\left(\Gamma_{j}\right) \\
& \leq C \sum_{j} \frac{M(\Delta)}{m(\Delta)} \ell_{\lambda}\left(\Gamma_{j}\right) \leq C^{2} \ell_{\lambda}(\Gamma),
\end{aligned}
$$

and (4.8) follows. Similarly by Lemma 4.7 Lemma 4.4 and the same estimate for $\sum_{j} \ell_{\lambda}\left(\Lambda_{j}\right)$,

$$
\begin{aligned}
|D \cap \Delta|_{\lambda} & \leq \sum_{j}\left|U_{j}\right|_{\lambda} \leq \sum_{j} C \ell_{\lambda}\left(\partial U_{j}\right) \\
& =C \sum_{j}\left[\ell_{\lambda}\left(\Lambda_{j}\right)+\ell_{\lambda}\left(\Gamma_{j}\right)\right] \leq\left(C^{3}+C\right) \ell_{\lambda}(\Gamma),
\end{aligned}
$$

and (4.9) is also proved.

Next let us consider the case $\beta \subseteq \bigcup_{j} \Lambda_{j}$. In this case, we have $\partial(D \cup \Delta) \subseteq \partial D \cup \beta$. In fact, if $x \in \partial(D \cup \Delta) \backslash \partial D$, then $x \in(\overline{D \cup \Delta})=\bar{D} \cup \Delta, x \notin(D \cup \Delta)^{\circ} \supseteq D \cup \Delta^{\circ}$ and $x \notin \bar{D} \backslash D$. In particular, $x \notin \bar{D} \backslash D$ and $x \notin D$, so $x \notin \bar{D}$. Hence $x \in \Delta$. But $x \notin \Delta^{\circ}$, so we must have $x \in \partial \Delta$; i.e., $x \in \partial \Delta \backslash \bar{D}=\beta$.

Then since $\beta \subseteq \bigcup_{j} \Lambda_{j}$, the estimate for $\sum_{j} \ell_{\lambda}\left(\Lambda_{j}\right)$ in (4.10) implies that

$$
\ell_{\lambda}(\partial(D \cup \Delta)) \leq \ell_{\lambda}(\partial D)+\ell_{\lambda}(\beta) \leq \ell_{\lambda}(\partial D)+C \ell_{\lambda}(\Gamma)
$$

for some $C=C(\epsilon)$. Also note that

$$
|D \cup \Delta|_{\lambda} \geq|D|_{\lambda} \geq|D|_{\lambda}-C \ell_{\lambda}(\Gamma) .
$$

Collecting (4.8), (4.9), (4.11) and (4.12), we have the following lemma.

Lemma 4.13. If $D$ is a Jordan region in $S$ and $\Delta$ a triangle in $\mathcal{T}$ such that $\Delta^{\circ} \cap \partial D \neq \emptyset$ and $D \nsubseteq \Delta$, then we may obtain a region $D^{\prime}$ by properly adding or subtracting $\Delta$ from $D$ so that the following properties hold for some constant $C=C(\epsilon)$ :

$$
\begin{aligned}
& \ell_{\lambda}\left(\partial D^{\prime}\right) \leq \ell_{\lambda}(\partial D)+C \ell_{\lambda}\left(\Delta^{\circ} \cap \partial D\right), \\
& |D|_{\lambda} \leq\left|D^{\prime}\right|_{\lambda}+C \ell_{\lambda}\left(\Delta^{\circ} \cap \partial D\right) .
\end{aligned}
$$

Note that $D$ does not have to be a Jordan region in Lemma4.13 The conclusion of this lemma is still valid under a weaker assumption that $D$ is open and $\Delta^{\circ} \cap \partial D$ is a countable disjoint union of Jordan $\operatorname{arcs}$ in $\Delta$ with endpoints on $\partial \Delta$.

The following theorem is due to L. Ahlfors.

Theorem 4.16 (Ahlfors). Let $S$ be an open simply-connected Riemann surface with a conformal metric $\lambda$. If $\lambda$ allows a linear isoperimetric inequality for every Jordan region in $S$, then $S$ is hyperbolic; i.e., conformally equivalent to the unit disc. 
For the proof of Theorem 4.16] see for example [14], pp. 143-144. In fact, this theorem is proved in [14] only when $\lambda$ is the pull-back spherical metric, but one can check that the same argument works when $\lambda$ is merely locally integrable.

Note that the surface $S$, when it is equipped with the metric $\lambda$ constructed in this section, does not have to be an Aleksandrov surface. However, one can see that Theorem 4.16 is still applicable to this case, because by (1.2) the pair $(S, \rho)$, where $\rho$ is the given metric which makes $S$ an Aleksandrov surface, can be identified with a Riemann surface and a conformal metric of the form (1.1), and $\lambda$ is conformally equivalent to $\rho$ by the construction.

Proof of Theorem 1.5. By Theorem 4.16, it suffices to show a linear isoperimetric inequality for every Jordan region $D$ in $S$. If $D$ is contained in a single triangle in $\mathcal{T}$ or is a union of triangles, then there is nothing to prove because of Lemma 4.4 or Corollary 4.6 respectively. So we assume that there exist a finite number of triangles $\Delta_{1}, \ldots, \Delta_{N}$ in $\mathcal{T}$ such that $\Delta_{j}^{\circ} \cap \partial D \neq \emptyset$ and $D \nsubseteq \Delta_{j}$ for $j=1, \ldots, N$. Since $\partial D$ is compact, there are only finitely many such triangles.

Let $D_{0}:=D$. Then we successively construct $D_{j}$ by either adding or subtracting $\Delta_{j}$ from $D_{j-1}$ so that (4.14) and (4.15) are satisfied. (The $D_{j}$ 's are not Jordan regions, but since $\Delta_{j}^{\circ}$ 's are disjoint we can still apply Lemma 4.13; see the statement following Lemma 4.13) By the construction, $D_{N}$ is a union of triangles in $\mathcal{T}$. Moreover we have $\Delta_{j}^{\circ} \cap \partial D_{j-1}=\Delta_{j}^{\circ} \cap \partial D$, because the $\Delta_{j}^{\circ}$ 's are disjoint. Therefore by Lemma 4.13 and Corollary 4.6 ,

$$
\begin{aligned}
|D|_{\lambda} & =\left|D_{0}\right|_{\lambda} \leq\left|D_{1}\right|_{\lambda}+C \ell_{\lambda}\left(\Delta_{1}^{\circ} \cap \partial D\right) \\
& \leq\left|D_{2}\right|_{\lambda}+C \ell_{\lambda}\left(\Delta_{2}^{\circ} \cap \partial D\right)+C \ell_{\lambda}\left(\Delta_{1}^{\circ} \cap \partial D\right) \\
& \leq \cdots \leq\left|D_{N}\right|+C \sum_{j=1}^{N} \ell_{\lambda}\left(\Delta_{j}^{\circ} \cap \partial D\right) \leq C \ell_{\lambda}\left(\partial D_{N}\right)+C \ell_{\lambda}(\partial D) \\
& \leq C\left[\ell_{\lambda}\left(\partial D_{N-1}\right)+C \ell_{\lambda}\left(\Delta_{N}^{\circ} \cap \partial D\right)\right]+C \ell_{\lambda}(\partial D) \\
& \leq C\left[\ell_{\lambda}\left(\partial D_{N-2}\right)+C \ell_{\lambda}\left(\Delta_{N-1}^{\circ} \cap \partial D\right)+C \ell_{\lambda}\left(\Delta_{N}^{\circ} \cap \partial D\right)\right]+C \ell_{\lambda}(\partial D) \\
& \leq \cdots \leq C \ell_{\lambda}\left(\partial D_{0}\right)+C^{2} \sum_{j=1}^{N} \ell_{\lambda}\left(\Delta_{j}^{\circ} \cap \partial D\right)+C \ell_{\lambda}(\partial D) \\
& \leq\left(C^{2}+2 C\right) \ell_{\lambda}(\partial D),
\end{aligned}
$$

where $C=C(\epsilon, M)$. This completes the proof.

Remark 4.17. Note that in the proof of Theorem 1.5 the assumption $S \in \mathfrak{S}(k)$ is never used globally - what we only used is the fact that every triangle $\Delta$ (or $\bar{\Delta}$ ) is a domain $R_{k}$. Also note that all constants in the proof of Theorem 1.5 are independent of $k$. (If we replace the assumption $(2 \pi-\epsilon) k^{-1 / 2}$ in (R2) by $2 \pi k^{-1 / 2}-\epsilon$, then the constant in Lemma 3.4 will depend on $k$.) From these observations, we deduce the following stronger result: suppose that an Aleksandrov surface $S$ has a tiling $\mathcal{T}$ and a partition $\mathcal{P}$ which satisfies all assumptions in Theorem 1.5 with $k=k(\Delta)<\infty$. Then $S$ is hyperbolic.

Remark 4.18. When $k>0$, suppose that we have

$(\mathrm{R} 1)^{\prime}$ (the length of every side of $\left.\Delta\right) \geq \epsilon$, for every $\Delta \in \mathcal{T}$

instead of (R1) in Theorem 1.5. Then one can see that our proof yields a linear isoperimetric inequality with respect to the original metric $\rho$ since $|\Delta|_{\rho} \leq 2 \pi k^{-1}$ 
and every side of a triangle $\Delta \in \mathcal{T}$ has length at most $\pi k^{-1 / 2}$. (One may also check that Lemma 3.4 is still valid in this case. In fact, (R1) is a result of $(\mathrm{R} 1)^{\prime}$ and (R2) when the curvature is constant $k>0$, which is exactly what we needed for the proof of Lemma 3.4.) When $k \leq 0$, the same is true using

$(\mathrm{R} 1)^{\prime \prime} \epsilon \leq$ (the length of every side of $\left.\Delta\right) \leq M$, for every $\Delta \in \mathcal{T}$.

Therefore in these cases the Aleksandrov surface satisfies a linear isoperimetric inequality with respect to $\rho$, hence it is also Gromov hyperbolic [13, [9], 17.

\section{Construction of Example 1.6}

For two given Riemann surfaces $\left(X_{i}, \pi_{i}\right), i=1,2$, spread over the complex plane, we use the notation $\left(X_{1}, \pi_{1}\right) \subseteq\left(X_{2}, \pi_{2}\right)$ if $X_{1} \subseteq X_{2}$ and $\left.\pi_{2}\right|_{X_{1}}=\pi_{1}$. A point $x \in X_{1}$ (or a set $A \subseteq X_{1}$ ) is called a point (or a set) over $y \in \mathbb{C}$ (or $B \subseteq \mathbb{C}$ ) if $\pi_{1}(x)=y$ (or $\pi_{1}(A)=B$, respectively).

In this section, we construct a sequence $\left(S_{1}, \varphi_{1}\right) \subseteq\left(S_{2}, \varphi_{2}\right) \subseteq \cdots$ of Riemann surfaces (with boundary) spread over proper subsets of $\mathbb{C}$, and the limit surface $(S, \varphi), S=\bigcup_{n=1}^{\infty} S_{n}$ and $\varphi=\lim _{n \rightarrow \infty} \varphi_{n}$ will serve as the surface described in Example 1.6. In fact, the surface $S$ equipped with the pull-back Euclidean metric, i.e., the metric whose length element is of the form $\left|\varphi^{\prime}(z) \| d z\right|$, will be the Aleksandrov surface satisfying all properties in Example 1.6

Before constructing this surface, however, we fix some more notations: we will always make the convention that each $S_{n}$ as well as each $S$ is equipped with the pullback Eulidean metric, and terminology such as shortest curves, area, distance, etc., will be used in this context. The symbol $|\cdot|$ will denote the area of a region in $S_{n}$ with respect to this metric, and $d(\cdot, \cdot)$ the distance between two points in $S_{n}$. Next, the term anticlockwise will be used for the preferred orientation of $\partial S_{n}$, where the orientation of $S_{n}$ is defined so that $\varphi_{n}$ is an orientation preserving map. If $\left(\Sigma_{n}, \tilde{\varphi}_{n}\right)$ is another Riemann surface spread over the plane such that $\left(S_{n}, \varphi_{n}\right) \subseteq\left(\Sigma_{n}, \tilde{\varphi}_{n}\right)$, then the term anticlockwise will also be used for $\partial \Sigma_{n}$ in the same way. Finally, for given $R>0, B(R)$ is the ball of radius $R$ in $\mathbb{C}$ with center at the origin, $\bar{B}(R)$ is its closure, and $C(R)$ is its boundary.

Now let us construct the example. We first take an equilateral triangle $\Delta_{0}$ inscribed in $C\left(R_{1}\right)$ for a sufficiently small $R_{1}>0$ such that $\left|\Delta_{0}\right| \leq \epsilon$. Let $S_{1}:=\Delta_{0}$, $\varphi_{1}:=$ identity and $\mathcal{T}_{1}:=\left\{\Delta_{0}\right\}$.

Suppose that we have constructed

$$
\left(S_{1}, \varphi_{1}\right) \subseteq\left(S_{2}, \varphi_{2}\right) \subseteq \cdots \subseteq\left(S_{n}, \varphi_{n}\right),
$$

their tilings $\mathcal{T}_{1} \subseteq \mathcal{T}_{2} \subseteq \cdots \subseteq \mathcal{T}_{n}$, and positive real numbers $R_{1} \leq R_{2} \leq \cdots \leq R_{n}$ which satisfy the following properties:

(a) each $\left(S_{i}, \varphi_{i}\right)$ is a surface spread over a proper subset of $\bar{B}\left(R_{i}\right)$ for $i=$ $1,2, \ldots, n$

(b) each $\mathcal{T}_{i}$ is a tiling of $S_{i}, i=1,2, \ldots, n$, consisting of finitely many triangles;

(c) each triangle in $\mathcal{T}_{n}$ has area at most $\epsilon$;

(d) all vertices of triangles in $\mathcal{T}_{n}$ lie over $\bigcup_{i=1}^{n} C\left(R_{i}\right)$;

(e) a vertex $v$ belongs to $\partial S_{i} \subseteq S_{n}$ if and only if $v$ is over $C\left(R_{i}\right), i=1,2, \ldots, n$;

(f) the set of critical points of $\varphi_{n}$ is equal to the set of all the vertices of triangles in $\mathcal{T}_{n-1}$, and at each critical point $\varphi_{n}$ has the local degree 2 . 
Let $t_{i}$ be the number of vertices of triangles in $\mathcal{T}_{n}$ which are over $C\left(R_{i}\right), i=$ $1,2, \ldots, n$, and choose $R_{n+1}$ such that

$$
R_{n+1} \geq R_{n} \exp \left\{2 \pi n \sum_{i=1}^{n} t_{i}+2 \pi n\right\}
$$

We then extend $\left(S_{n}, \varphi_{n}\right)$ to a branched covering $\left(\Sigma_{n}, \tilde{\varphi}_{n}\right)$ of $\bar{B}\left(R_{n+1}\right)$ so that $\left(S_{n}, \varphi_{n}\right) \subseteq\left(\Sigma_{n}, \tilde{\varphi}_{n}\right)$ and the set of critical points of $\tilde{\varphi}_{n}$ is equal to that of $\varphi_{n}$. For example, $\Sigma_{1}=\bar{B}\left(R_{2}\right)$ and $\tilde{\varphi}_{1}=$ identity since $\varphi_{1}$ has no singular point, and $\tilde{\varphi}_{2}: \Sigma_{2} \rightarrow \bar{B}\left(R_{3}\right)$ is a four-sheeted branched covering with critical points of the local degree 2 at the vertices of $\Delta_{0}$. Similarly one can check by the Riemann-Hurwitz formula that $\tilde{\varphi}_{n}: \Sigma_{n} \rightarrow \bar{B}\left(R_{n+1}\right)$ is a $\left(\sum_{i=1}^{n-1} t_{i}+1\right)$-sheeted branched covering. Now let $\left\{v_{1}, v_{2}, \ldots, v_{t_{n}}\right\}$ be an anticlockwise enumeration (modulo $t_{n}$ ) of the vertices of triangles in $\mathcal{T}_{n}$ which belong to $\partial S_{n}$. Then choose $t_{n}$ points $w_{1}, w_{2}, \ldots, w_{t_{n}}$ on $\partial \Sigma_{n}$, also enumerated anticlockwise in modulo $t_{n}$, so that $\left|\Delta_{n}^{j}\right| \leq \epsilon$, where $\Delta_{n}^{j}$ is the triangle in $\Sigma_{n}$ with vertices $v_{j}, v_{j+1}$ and $w_{j+1}$. This is possible by making the angle of $\Delta_{n}^{j}$ at $v_{j+1}$ appropriately small. (See Figure 1.)

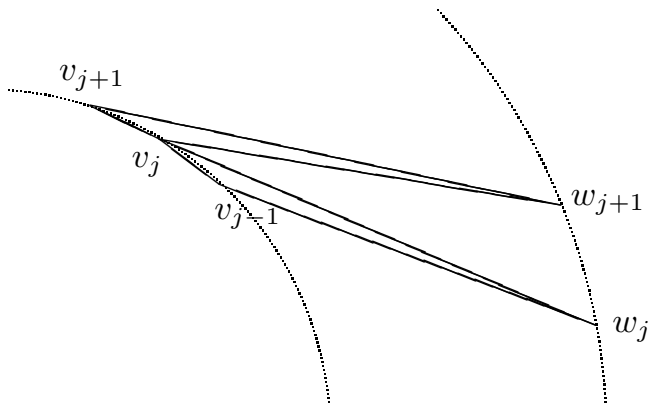

Figure 1

Now for each $j=1,2, \ldots, t_{n}$, let $\Gamma_{j}$ be a copy of $\bar{B}\left(R_{n+1}\right)$ and let $\pi_{j}: \Gamma_{j} \rightarrow$ $\bar{B}\left(R_{n+1}\right)$ be the identity map. On each $\Gamma_{j}$, we mark two points $v^{j}$ and $w^{j}$ so that $\pi_{j}\left(v^{j}\right)=\tilde{\varphi}_{n}\left(v_{j}\right)$ and $\pi_{j}\left(w^{j}\right)=\tilde{\varphi}_{n}\left(w_{j}\right)$. Then for each $j$, we cut $\Gamma_{j}$ along the shortest curve $\left[v^{j}, w^{j}\right]$, and cut $\Sigma_{n}$ along the shortest curves $\left[v_{j}, w_{j}\right]$ for all $j=1,2, \ldots, t_{n}$. Now we glue each $\Gamma_{j}$ to $\Sigma_{n}, j=1,2, \ldots, t_{n}$, along the corresponding cuts. This is possible since $\pi_{j}\left(\left[v^{j}, w^{j}\right]\right)=\tilde{\varphi}_{n}\left(\left[v_{j}, w_{j}\right]\right)$ for all $j$, and in this way we have a new Riemann surface $\left(\Sigma_{n}^{\prime}, \tilde{\varphi}_{n}^{\prime}\right)$ spread over $\bar{B}\left(R_{n+1}\right)$.

We transfer notations such as $w_{j}, v_{j}$, etc., to $\Sigma_{n}^{\prime}$ in the following way: each of $w_{j} \in \partial \Sigma_{n}$ and $w^{j} \in \partial \Gamma_{j}$ corresponds to two points in $\partial \Sigma_{n}^{\prime}$, which are also denoted by $w_{j}$ and $w^{j}$; we place them in the order $\ldots, w_{j}, w^{j}, w_{j+1}, \ldots$ anticlockwise; each of $v_{j} \in \Sigma_{n}$ and $v^{j} \in \Gamma_{j}$ corresponds to only one point in $\Sigma_{n}^{\prime}$, which is denoted by $v_{j} ; \Delta_{n}^{j}$ is regarded as a triangle in $\Sigma_{n}^{\prime}$ as well as in $\Sigma_{n}$ with vertices $v_{j}, v_{j+1}$ and $w_{j+1}$.

To complete the construction of $\left(S_{n+1}, \varphi_{n+1}\right)$, let $M_{n}$ be a positive integer which will be determined later, and choose a set of points

$$
\left\{w_{j}^{k}: 1 \leq j \leq t_{n}, 0 \leq k \leq M_{n}\right\}
$$


on $\partial \Sigma_{n}^{\prime}$, where $w_{j}^{k}$ 's are enumerated anticlockwise for fixed $j$, so that for all $j$ and $k, w_{j}^{0}=w_{j}=w_{j-1}^{M_{n}}$ and $d\left(w_{j}^{k}, w_{j}^{k+1}\right)=d\left(w_{j}^{k-1}, w_{j}^{k}\right)$. Let $\Delta_{n}^{j k}$ be the triangle in $\Sigma_{n}^{\prime}$ with vertices $w_{j}^{k}, w_{j}^{k+1}$ and $v_{j}$ for $1 \leq j \leq t_{n}$ and $0 \leq k \leq M_{n}-1$. Taking $M_{n}$ sufficiently large, we have $\left|\Delta_{n}^{j k}\right| \leq \epsilon$. Then the set

$$
\mathcal{T}_{n+1}:=\mathcal{T}_{n} \cup\left\{\Delta_{n}^{j}: 1 \leq j \leq t_{n}\right\} \cup\left\{\Delta_{n}^{j k}: 1 \leq j \leq t_{n}, 0 \leq k \leq M_{n}-1\right\}
$$

is a tiling of $S_{n+1}:=\bigcup_{\Delta \in \mathcal{T}_{n+1}} \Delta$, and we define

$$
\varphi_{n+1}=\left.\tilde{\varphi}_{n}^{\prime}\right|_{S_{n+1}} \text {. }
$$

Note that $S_{n+1}$ is obtained from $\Sigma_{n}^{\prime}$ by cutting off some parts outside triangles in $\mathcal{T}_{n+1}$, and $\left(S_{n+1}, \varphi_{n+1}\right), \mathcal{T}_{n+1}$ and $R_{n+1}$ satisfy all properties (a)-(f) above.

Now let $S:=\bigcup_{n=1}^{\infty} S_{n}=\bigcup_{n=1}^{\infty} \Sigma_{n}^{\prime}, \varphi=\lim _{n \rightarrow \infty} \varphi_{n}$ and $\mathcal{T}:=\bigcup_{n=1}^{\infty} \mathcal{T}_{n}$. Clearly the pair $(S, \varphi)$ is a Riemann surface spread over the plane with the tiling $\mathcal{T}$. Since each $S_{n}$ is simply connected, so is $S$. Furthermore, the area of all triangles in $\mathcal{T}$ is at most $\epsilon$ by (c) and each vertex has total angle $4 \pi$ by (f). Hence it remains to show that $S$ is conformally equivalent to $\mathbb{C}$, i.e., parabolic.

Let $B_{n}:=B\left(R_{n+1}\right) \backslash \bar{B}\left(R_{n}\right) \subseteq \mathbb{C}$ and $A_{n}:=\varphi^{-1}\left(B_{n}\right) \cap \Sigma_{n}^{\prime}$. Then one can easily see that $\left\{A_{n}\right\}$ is a sequence of concentric annuli in $S$. Moreover, since each $\Sigma_{n}^{\prime}$ is a $\left(\sum_{i=1}^{n} t_{i}+1\right)$-sheeted branched covering of $\bar{B}\left(R_{n+1}\right)$ and there is no critical point of $\varphi$ over $B_{n}$, each $A_{n}$ is a $\left(\sum_{i=1}^{n} t_{i}+1\right)$-sheeted unbranched covering of $B_{n}$. Then because the module (cf. [1] or [21] ) of $B_{n}$ is $(2 \pi)^{-1} \log \left(R_{n+1} / R_{n}\right)$, the module of $A_{n}$ is

$$
\frac{1}{2 \pi\left(\sum_{i=1}^{n} t_{i}+1\right)} \log \frac{R_{n+1}}{R_{n}}
$$

which is greater than or equal to $n$ by (5.1). So the module of $A_{n}$ tends to $\infty$ as $n \rightarrow \infty$, and this shows that $S$ is parabolic. In fact, a comparison theorem (1], p. 54) implies that the module of each $A_{n}$ is less than or equal to that of the unbounded component of $S \backslash A_{1}$, which should be finite if $S$ were hyperbolic.

\section{Line COMPLEXES AND HYPERBOLICITY}

Suppose that $(S, \varphi)$ is a simply-connected Riemann surface spread over the sphere such that for some $q \geq 2$ points $a_{1}, \ldots, a_{q} \in \overline{\mathbb{C}}$, the restriction map

$$
\varphi: S \backslash\left\{\varphi^{-1}\left(a_{j}\right): j=1,2, \ldots, q\right\} \rightarrow \overline{\mathbb{C}} \backslash\left\{a_{1}, \ldots, a_{q}\right\}
$$

is a topological covering map. This pair $(S, \varphi)$ is called a Riemann surface of class $F_{q}$ ramified over the points $a_{1}, \ldots, a_{q}$, and denoted by $(S, \varphi) \in F_{q}\left(a_{1}, \ldots, a_{q}\right)$, or $S \in F_{q}$ if there is no confusion.

Now we draw through the points $a_{\nu}$ in the order $a_{1}, \ldots, a_{q}$, with $a_{1}$ a Jordan curve $\gamma$, called a base curve, decomposing $\overline{\mathbb{C}}$ into two simply-connected open regions $G_{1}$ and $G_{2}$, where $G_{1}$ is on the left of $\gamma$. These "half sheets" $G_{1}$ and $G_{2}$ are called polygons for short, $a_{1}, \ldots, a_{q}$ vertices, and the subarcs of $\gamma,\left(a_{1} a_{2}\right),\left(a_{2} a_{3}\right), \ldots$, $\left(a_{q} a_{1}\right)$, sides of the polygons. Next we choose two points $\circ \in G_{1}$ and $\times \in G_{2}$, and for each $j=1,2, \ldots, q$, let $\gamma_{j}$ be a simple arc joining these two points and crossing the side $\left(a_{j} a_{j+1}\right)$ at exactly one point so that $\gamma_{j} \cap \gamma_{k}=\{0, \times\}$ for $i \neq k$. Let $\Gamma_{0}$ be the graph in $\overline{\mathbb{C}}$ consisting of $\circ, \times$ and $\gamma_{j}$ 's. Then the pull-back graph $\Gamma:=\varphi^{-1}\left(\Gamma_{0}\right)$ is a planar graph which is properly embedded in $\mathbb{C}$, and its homeomorphic equivalence 4

\footnotetext{
${ }^{4}$ Two properly embedded planar graphs $\Gamma_{1}$ and $\Gamma_{2}$ are called homeomorphically equivalent if there is a homeomorphism $h: \mathbb{C} \rightarrow \mathbb{C}$ such that $h\left(\Gamma_{1}\right)=\Gamma_{2}$.
} 
class is called the line complex or the Speiser graph of degree $q$ corresponding to $S \in F_{q}$ ([16, Chap. XI). The components of $\mathbb{C} \backslash \Gamma$ will be called faces of $\Gamma$, and $\Gamma$ has the following properties:

(i) $\Gamma$ is connected;

(ii) $\Gamma$ is bipartite, i.e., all vertices are split into two disjoint subsets, say $V_{\circ}:=$ $\varphi^{-1}(\circ)$ and $V_{\times}:=\varphi^{-1}(\times)$, and every edge joins a point in $V_{\circ}$ and a point in $V_{\times}$;

(iii) every vertex has the same degree $q$, i.e., each vertex corresponds to $q$ different edges;

(iv) the edges can be labeled by $1,2, \ldots, q$ so that they are placed counterclockwise around the points in $V_{\circ}$ and clockwise around the points in $V_{\times}$.

Conversely, suppose we are given a line complex $\Gamma$ of degree $q$, i.e., suppose $\Gamma$ is (a homeomorphic equivalence class of) a planar graph which is properly embedded in $\mathbb{C}$ and satisfies (i)-(iv). Then for a fixed base curve $\gamma$ passing through $a_{1}, \ldots, a_{q}$ in this order, there exists a unique $S \in F_{q}$ whose corresponding line complex is $\Gamma$. Therefore for fixed base points $a_{1}, \ldots, a_{q}$ and a base curve $\gamma$, there is a one-to-one correspondence between Riemann surfaces of class $F_{q}$ and line complexes of degree $q$.

Each vertex $p$ of $\Gamma$ corresponds to a half sheet - a connected component of $\varphi^{-1}\left(G_{1}\right)$ or $\varphi^{-1}\left(G_{2}\right)$ - of $S$, and each face of $\Gamma$ corresponds to a singularity of $\varphi$. In fact, each face is a $2 m$-gon for some $m \in \mathbb{N} \cup\{\infty\}$, and if $m<\infty$ the face of $\Gamma$ corresponds to a point in $S$ at which $\varphi$ has the local degree $m$, and if $m=\infty$ the face corresponds to a logarithmic singularity of $\varphi$.

The following definition is due to R. Nevanlinna.

Definition 6.2. Let $p$ be a vertex of $\Gamma$ and let $f_{1}, \ldots, f_{q}$ be the faces of $\Gamma$ with $p$ on their boundaries. If each face $f_{i}$ is a $2 m_{i}$-gon for $i=1, \ldots, q$, the excess $E_{p}$ of $\Gamma$ at $p$ is defined as

$$
E_{p}:=\sum_{i=1}^{q} \frac{1}{m_{i}}-q+2 .
$$

We interpret $1 / m_{i}=0$ if $m_{i}=\infty$ for some $i$.

A Riemann surface $S$ of class $F_{q}$ is called regularly ramified if there is a real number $E$ such that $E=E_{p}$ for every vertex $p$ of the corresponding line complex $\Gamma$.

Theorem 6.4 (Nevanlinna [16]). Suppose that $S$ is a regularly ramified open Riemann surface of class $F_{q}$. Then $S$ is parabolic if $E=0$ and hyperbolic if $E<0$.

Our purpose in this section is to generalize the hyperbolic case $E<0$ of this theorem. Also note that the case $E>0$ happens only when $S$ is compact, i.e., $S=\overline{\mathbb{C}}$.

Suppose that $\Gamma_{\alpha}$ is a connected subgraph of $\Gamma$ and $V_{\alpha} \subseteq V_{\circ} \cup V_{\times}$the vertex set of $\Gamma_{\alpha}$. We then identify $\Gamma_{\alpha}$ with $V_{\alpha}$, and terminology from the set theory such as union, intersection, disjoint, etc., will be used for subgraphs in this context. A set $\mathcal{P}(\Gamma)$ of subgraphs is called a partition of $\Gamma$ if each element $\Gamma_{\alpha} \in \mathcal{P}(\Gamma)$ is connected as a subgraph of $\Gamma$, the elements in $\mathcal{P}(\Gamma)$ are disjoint, and $\bigcup_{\Gamma_{\alpha} \in \mathcal{P}(\Gamma)} \Gamma_{\alpha}=\Gamma$. Let $\#\left(\Gamma_{\alpha}\right)$ be the number of vertices in $\Gamma_{\alpha}$. 
Theorem 6.5. Suppose that $\Gamma$ is a line complex with the associated Riemann surface $S$ of class $F_{q}$. If $\Gamma$ has a partition $\mathcal{P}(\Gamma)$ such that for some constants $\epsilon>0$ and $M>0$,

(M1)' $\#\left(\Gamma_{\alpha}\right) \leq M$ for every $\Gamma_{\alpha} \in \mathcal{P}(\Gamma)$,

$(\mathrm{M} 2)^{\prime} \sum_{p \in \Gamma_{\alpha}} E_{p} \leq-\epsilon$ for every $\Gamma_{\alpha} \in \mathcal{P}(\Gamma)$,

then $S$ is hyperbolic.

Proof. Because the surface $S$ equipped with the pull-back spherical metric is in $\mathfrak{S}(1)$ (cf. Section 2.4), it suffices to construct a tiling $\mathcal{T}$ and a partition $\mathcal{P}$ which satisfy the four conditions in Theorem 1.5

Without loss of generality, we assume that the base curve $\gamma$ is a union of a finite number $(\geq q)$ of geodesic line segments in $\overline{\mathbb{C}}$. Furthermore, we may assume that the angle of $\gamma$ at each $a_{j}$ is $\pi$ for all $j=1, \ldots, q$, and that the two components $G_{1}$ and $G_{2}$ of $\overline{\mathbb{C}} \backslash \gamma$ have the same areas $2 \pi$. We then construct a finite tiling $\mathcal{T}^{\prime}$ of $\overline{\mathbb{C}}$ so that the vertex set of triangles in $\mathcal{T}^{\prime}$ contains all $a_{j}$ 's and all the endpoints of geodesic line segments in $\gamma$, and that both $G_{1}$ and $G_{2}$ are unions of a finite number of triangles in $\mathcal{T}^{\prime}$.

The tiling $\mathcal{T}^{\prime}$ of $\overline{\mathbb{C}}$ induces a tiling $\mathcal{T}$ of $S$ via the map $\varphi: S \rightarrow \overline{\mathbb{C}}$ as follows: for each $\Delta^{\prime} \in \mathcal{T}^{\prime}$, the closure of each component of $\varphi^{-1}\left(\Delta^{\circ}\right)$ is a triangle $\Delta \in S$ satisfying the conditions in Definition 2.3, hence we let $\mathcal{T}$ be the collection of such triangles. One can easily check that $\mathcal{T}$ satisfies the definition of a tiling given in the Introduction, and note that the $\bar{S}$-closure $\bar{\Delta}$ of $\Delta$ is isometric to $\Delta^{\prime}$. Similarly, the partition $\mathcal{P}(\Gamma)$ of $\Gamma$ induces a partition $\mathcal{P}$ of $\mathcal{T}$. In fact, each vertex $p$ of $\Gamma$ corresponds to a half sheet - a connected component of $\varphi^{-1}\left(G_{1}\right)$ or $\varphi^{-1}\left(G_{2}\right)$. Hence each subgraph $\Gamma_{\alpha} \in \mathcal{P}(\Gamma)$ corresponds to a union of half sheets, say $C_{\alpha}$, which is connected since each $\Gamma_{\alpha}$ is connected. Finally, since both $G_{1}$ and $G_{2}$ consist of triangles in $\mathcal{T}^{\prime}$, each $C_{\alpha}$ consists of triangles in $\mathcal{T}$. Therefore $C_{\alpha}$ is a cluster. Now one can easily check that all the other assumptions about $\mathcal{P}$ follow from the assumptions about $\mathcal{P}(\Gamma)$.

We verify that the tiling $\mathcal{T}$ and the partition $\mathcal{P}$ defined above satisfy all the conditions of Theorem 1.5. First, conditions (R1) and (R2) are trivially satisfied because for every $\Delta \in \mathcal{T}$, the $\bar{S}$-closure $\bar{\Delta}$ of $\Delta$ is isometric to a triangle $\Delta^{\prime}$ in $\mathcal{T}^{\prime}$, and $\mathcal{T}^{\prime}$ contains only finitely many triangles. Furthermore (M1)' implies (M1) since there are only finitely many triangles in both $G_{1}$ and $G_{2}$.

It remains to show that condition (M2) holds. To do this, fix a vertex $p$ of $\Gamma$, and let $H$ be the corresponding half sheet of $S$, let $f_{1}, \ldots, f_{q}$ be the faces of $\Gamma$ with $p$ on their boundary, and let $\nu_{1}, \ldots, \nu_{q}$ be the corresponding singularities of $S$ lying on the $\bar{S}$-boundary $\partial H$ of $H$. If $f_{j}$ is $2 m_{j}$-gon for $j=1,2, \ldots, q$, we have $T\left(\nu_{j}\right)=2 \pi m_{j}$. Moreover, since the angle of $\gamma$ at $a_{j}$ is $\pi$, the angle of $\partial H$ at $\nu_{j}$ is also $\pi$. Finally, we have $T(v)=2 \pi$ for every vertex $v \neq \nu_{j}, j=1,2, \ldots, q$, of triangles in $H$. Therefore,

$$
\begin{array}{r}
\sum_{\Delta \subseteq H} \sum_{i=1}^{3} \frac{2 \pi-T\left(v_{i}(\Delta)\right)}{T\left(v_{i}(\Delta)\right)} \theta\left(v_{i}(\Delta), \Delta\right)=\sum_{j=1}^{q} \sum_{\Delta \subseteq H} \frac{2 \pi-T\left(\nu_{j}\right)}{T\left(\nu_{j}\right)} \theta\left(\nu_{j}, \Delta\right) \\
\quad=\sum_{j=1}^{q} \frac{2 \pi-T\left(\nu_{j}\right)}{T\left(\nu_{j}\right)} \cdot \pi=\sum_{j=1}^{q} \frac{2 \pi-2 \pi m_{j}}{2 \pi m_{j}} \cdot \pi=\pi\left(\sum_{j=1}^{q} \frac{1}{m_{j}}-q\right)
\end{array}
$$


where the $v_{i}(\Delta)$ 's are the vertices of $\Delta$. Here we used the convention that $\theta(v, \Delta)=$ 0 if $v$ is not a vertex of $\Delta$, and that $\infty / \infty=1$ and $a / \infty=0$ for $a<\infty$.

On the other hand, the Gaussian curvature of the pull-back spherical metric on $S$ exists and is 1 at non-singular points. Hence if $D$ is a region in $S$ which contains no singular points, the integral curvature $\omega(D)$ of $D$ is same as the area of $D$. Since $H$ contains no singular point in its interior and is isometric to either $G_{1}$ or $G_{2}$, we have

$$
\sum_{\Delta \subseteq H} \omega\left(\Delta^{\circ}\right)=\omega\left(H^{\circ}\right)=2 \pi .
$$

Also note that the left turn of each side of triangles in $\mathcal{T}$ is zero because it is a locally geodesic curve containing no singular points on its interior arc. Therefore by the discussion in Section 2.3 we have

$$
K(\Delta)=\sum_{i=1}^{3} \frac{2 \pi-T\left(v_{i}\right)}{T\left(v_{i}\right)} \theta\left(v_{i}, \Delta\right)+\omega\left(\Delta^{\circ}\right),
$$

and by (6.6), (6.7) and (6.8) we conclude that

$$
\begin{gathered}
\sum_{\Delta \subseteq H} K(\Delta)=\sum_{\Delta \subseteq H}\left(\sum_{i=1}^{3} \frac{2 \pi-T\left(v_{i}(\Delta)\right)}{T\left(v_{i}(\Delta)\right)} \theta\left(v_{i}(\Delta), \Delta\right)+\omega\left(\Delta^{\circ}\right)\right) \\
=\sum_{\Delta \subseteq H} \sum_{i=1}^{3} \frac{2 \pi-T\left(v_{i}(\Delta)\right)}{T\left(v_{i}(\Delta)\right)} \theta\left(v_{i}(\Delta), \Delta\right)+\sum_{\Delta \subseteq H} \omega\left(\Delta^{\circ}\right) \\
=\pi\left(\sum_{j=1}^{q} \frac{1}{m_{j}}-q\right)+2 \pi=\pi\left(\sum_{j=1}^{q} \frac{1}{m_{j}}-q+2\right)=\pi E_{p} .
\end{gathered}
$$

Now it is easy to see that (M2)' implies (M2). The theorem follows.

This proof, as we discussed in Remark 4.18, in fact shows that the surface $S \in F_{q}$ satisfies a linear isoperimetric inequality with respect to the pull-back spherical metric.

Remark 6.9. Theorem 6.5 can be regarded as a generalization of the hyperbolic case of Theorem 6.4. First of all, Theorem 6.4 is nothing but the case $M=1$ in Theorem 6.5. Second, $S$ does not have to be regularly ramified in Theorem 6.5 while it has to be in Theorem 6.4 Also note that some points over $a_{j}$ 's are even allowed to be unramified in Theorem 6.5 .

We finish this paper with some discussion about Nevanlinna's conjecture. Suppose a line complex $\Gamma$ of degree $q$ and the corresponding Riemann surface $S$ of class $F_{q}$ are given. Let $V \Gamma$ be the vertex set of $\Gamma$ and define the distance $d_{\Gamma}\left(p, p^{\prime}\right)$ between two vertices $p, p^{\prime} \in V \Gamma$ as the infimum of the (combinatorial) lengths of curves in $\Gamma$ connecting $p$ and $p^{\prime}$. Now for a vertex $p \in V \Gamma$, let

$$
B(p, j):=\left\{p^{\prime} \in V \Gamma: d_{\Gamma}\left(p, p^{\prime}\right) \leq j\right\} .
$$

R. Nevanlinna defined the mean excess of $\Gamma$ (or $S$ ) by the formula

$$
E:=\lim _{j \rightarrow \infty} \frac{1}{n_{j}} \sum_{p^{\prime} \in B(p, j)} E_{p^{\prime}},
$$


where $n_{j}$ is the number of vertices in $B(p, j)$. If this limit does not exist, one can only consider the upper $(\bar{E})$ or lower $(\underline{E})$ excess of $S$, which are defined by (6.10) with limit superior or limit inferior in place of the limit, respectively. Note that this definition of excess coincides with the quantity $E$ in Theorem 6.4 when $S$ is regularly ramified. In general $E$ depends on the base point $p$.

If $S$ is an $n$-sheeted closed surface, i.e., the corresponding line complex is a finite graph with $2 n$ vertices, one can show by Euler's formula that $E$ always exists and is equal to $2 / n$. For an infinite-sheeted open surface $S$, Nevanlinna stated the following conjecture [16]: Is the surface parabolic if $E=0$ and hyperbolic if $E<0$ ? Unfortunately, however, the answer is negative. Teichmüller [20] constructed a hyperbolic surface with $E=0$ and recently I. Benjamini, S. Merenkov and O. Schramm [5] proved the existence of a parabolic surface with $\bar{E}<0$.

Therefore a stronger condition is necessary. Indeed, we prove the following Theorem 6.11 with an additional "uniform" type assumption.

Theorem 6.11. Suppose $\Gamma$ is a line complex and let $S$ be the associated Riemann surface of class $F_{q}$. If there exist constants $\epsilon>0$ and $M>0$ such that

$$
\sum_{p \in \Gamma_{0}} E_{p} \leq-\epsilon
$$

for every finite connected subgraph $\Gamma_{0}$ with $\#\left(\Gamma_{0}\right) \geq M$, then $S$ is hyperbolic.

We need the following two lemmas for the proof of this theorem.

Lemma 6.12. Suppose $\Lambda$ is a connected subgraph of a line complex of degree $q$ such that $4 q \leq \#(\Lambda)=: K<\infty$. Then there are two disjoint connected subgraphs $\Lambda_{1}$ and $\Lambda_{2}$ of $\Lambda$ such that $\Lambda_{1} \cup \Lambda_{2}=\Lambda$ and $\#\left(\Lambda_{i}\right) \geq K / 2 q$ for $i=1,2$.

Proof. We describe an algorithm to find such $\Lambda_{1}$ and $\Lambda_{2}$.

Step 1. Take a subgraph $\Lambda^{1} \subseteq \Lambda$ such that $\#\left(\Lambda^{1}\right)=1$. If $\Lambda \backslash \Lambda^{1}$ is disconnected, we set $\Lambda^{0}=\emptyset$ and go to Step 3 with $k=1$. Otherwise, we go to Step 2 with $k=2$.

Step 2. Suppose that we have found a connected subgraph $\Lambda^{k-1}$ such that $\Lambda \backslash \Lambda^{k-1}$ is connected and $\#\left(\Lambda^{k-1}\right)=k-1<K / 2 q$. We then choose a connected subgraph $\Lambda^{k}$ such that $\Lambda^{k-1} \subseteq \Lambda^{k} \subseteq \Lambda$ and $\#\left(\Lambda^{k}\right)=k$. If $\Lambda \backslash \Lambda^{k}$ is disconnected, we go to Step 3. If $\Lambda \backslash \Lambda^{k}$ is connected and $k=K / 2 q$, then the lemma follows with $\Lambda_{1}=\Lambda^{k}$ and $\Lambda_{2}=\Lambda \backslash \Lambda^{k}$. If $\Lambda \backslash \Lambda^{k}$ is connected and $k<K / 2 q$, then we repeat Step 2 .

Step 3. Let $p$ be the vertex of $\Lambda$ such that $\{p\}=\Lambda^{k} \backslash \Lambda^{k-1}$. Then since $\Lambda \backslash \Lambda^{k-1}$ is connected and there are at most $q$ edges at $p$, there are at most $q$ components in $\Lambda \backslash \Lambda^{k}$. Moreover because $k<K / 2 q+1 \leq K / 2$,

$$
\#\left(\Lambda \backslash \Lambda^{k}\right)=K-k \geq \frac{K}{2},
$$

hence there exists a component $\Lambda_{1}$ such that $\#\left(\Lambda_{1}\right) \geq K / 2 q$. Let $\Lambda_{2}:=\Lambda \backslash \Lambda_{1}$. The subgraph $\Lambda_{2}$ is connected because each component in $\Lambda \backslash \Lambda^{k}$ is connected by an edge to $p \in \Lambda^{k}$. Now there are two cases. If $\#\left(\Lambda_{2}\right) \geq K / 2 q$, then $\Lambda_{1}$ and $\Lambda_{2}$ satisfy all the properties in the lemma. If $\#\left(\Lambda_{2}\right)<K / 2 q$, let $\Lambda^{k^{\prime}-1}:=\Lambda_{2}$ where $k^{\prime}-1=\#\left(\Lambda_{2}\right)$, and note that $k+1 \leq k^{\prime}$ because $\Lambda^{k} \subseteq \Lambda_{2}$ by our construction. Now we repeat Step 2 with $k^{\prime}=k$. This completes the steps.

Note that this process must terminate since $K<\infty$. The lemma follows. 
Lemma 6.13. Suppose $\Lambda$ is a connected subgraph of a line complex of degree $q$ such that $\#(\Lambda)=\infty$. Then for any integer $M \geq 2$, there are disjoint connected subgraphs $\Lambda_{1}, \Lambda_{2}, \ldots, \Lambda_{s}$ for some $s$ such that $\bigcup_{i=1}^{s} \Lambda_{i}=\Lambda$, \#( $\left.\Lambda_{1}\right)<\infty$, and for each $i$, either $\#\left(\Lambda_{i}\right)=\infty$ or $M \leq \#\left(\Lambda_{i}\right) \leq 2 q M^{2}$.

Proof. We first take a connected subgraph $\Lambda_{1} \subseteq \Lambda$ such that $\#\left(\Lambda_{1}\right)=M$. Since each component of $\Lambda \backslash \Lambda_{1}$ is connected by an edge to at least one vertex of $\Lambda_{1}$ and each vertex has at most $q$ edges, $\Lambda \backslash \Lambda_{1}$ has at most $q M$ components. Let $\Lambda^{1}, \Lambda^{2}, \ldots, \Lambda^{k}, k \leq q M$, be the components of $\Lambda \backslash \Lambda_{1}$.

If $\#\left(\Lambda^{j}\right)=\infty$ or $M \leq \#\left(\Lambda^{j}\right) \leq 2 q M$ for some $j$ 's, we take each of these $\Lambda^{j}$ 's as one of $\Lambda_{i}, i \geq 2$, in the lemma. If $2 q M<\#\left(\Lambda^{j}\right)<\infty$ for some $j$ 's, we apply Lemma 6.12 to each of these and their subgraphs repeatedly to find disjoint connected subgraphs $\Lambda^{j 1}, \Lambda^{j 2}, \ldots, \Lambda^{j m_{j}}$ such that $M \leq \#\left(\Lambda^{j l}\right) \leq 2 q M$, $l=1, \ldots, m_{j}$, and $\bigcup_{l=1}^{m_{j}} \Lambda^{j l}=\Lambda^{j}$. We then take each of these $\Lambda^{j l}$ 's as one of $\Lambda_{i}$, $i \geq 2$. Finally, we replace $\Lambda_{1}$ by $\Lambda_{1} \cup \cup \Lambda^{j}$, where the union is over all $\Lambda^{j}$ 's with $\#\left(\Lambda^{j}\right)<M$. Note that $\Lambda_{1}$ remains to be connected and $\#\left(\Lambda_{1}\right) \leq 2 q M^{2}$ since $k$, the number of components in $\Lambda \backslash \Lambda_{1}$, is at most $q M$. This completes the proof.

Proof of Theorem 6.11. It is enough to find a partition $\mathcal{P}(\Gamma)$ of $\Gamma$ satisfying

$$
M \leq \#\left(\Gamma_{\alpha}\right) \leq 2 q M^{2}
$$

for all $\Gamma_{\alpha} \in \mathcal{P}(\Gamma)$. Conditions (M1) ${ }^{\prime}$ and (M2) in Theorem6.5 are trivially satisfied in this case.

Let $\mathcal{F} \subseteq 2^{\Gamma}$ be the set of all connected subgraphs of $\Gamma$. Then we define

$$
\begin{aligned}
\mathfrak{P}:= & \left\{P \subseteq \mathcal{F}: \Gamma \backslash \bigcup_{\Gamma_{\alpha} \in P} \Gamma_{\alpha} \text { has no finite component, and for all } \Gamma_{\alpha}\right. \\
& \text { and } \left.\Gamma_{\beta} \text { in } P, M \leq \#\left(\Gamma_{\alpha}\right) \leq 2 q M^{2} \text { and } \Gamma_{\alpha} \cap \Gamma_{\beta}=\emptyset \text { if } \alpha \neq \beta\right\} .
\end{aligned}
$$

The collection $\mathfrak{P}$ is non-empty since $\emptyset \in \mathfrak{P}$, and $\mathfrak{P}$ is partially ordered under usual set inclusion. Moreover, every chain $P_{1} \subseteq P_{2} \subseteq \cdots$ in $\mathfrak{P}$ has an upper bound $P^{\prime}=\bigcup P_{i}$. In fact, if $\Lambda$ is a finite component of $\Gamma \backslash \bigcup_{\Gamma_{\alpha} \in P^{\prime}} \Gamma_{\alpha}$, then it is connected by single edges to a finite number of vertices in $\bigcup_{\Gamma_{\alpha} \in P^{\prime}} \Gamma_{\alpha}$. But all of these vertices must belong to $\bigcup_{\Gamma_{\alpha} \in P_{n}} \Gamma_{\alpha}$ for sufficiently large $n$, so $\Lambda$ is a finite component of $\Gamma \backslash \bigcup_{\Gamma_{\alpha} \in P_{n}} \Gamma_{\alpha}$. This is impossible because $P_{n} \in \mathfrak{P}$, and one can easily see that $P^{\prime} \in \mathfrak{P}$.

Now Zorn's lemma implies the existence of a maximal element $\mathcal{P}(\Gamma)$ in $\mathfrak{P}$. To show that $\mathcal{P}(\Gamma)$ is a desired partition, it suffices to show $\bigcup_{\Gamma_{\alpha} \in \mathcal{P}(\Gamma)} \Gamma_{\alpha}=\Gamma$. But if $\bigcup_{\Gamma_{\alpha} \in \mathcal{P}(\Gamma)} \Gamma_{\alpha} \neq \Gamma$, then there is a component $\Lambda$ in $\Gamma \backslash \bigcup_{\Gamma_{\alpha} \in \mathcal{P}(\Gamma)} \Gamma_{\alpha}$, which must be an infinite component by the definition of $\mathfrak{P}$. We then apply Lemma 6.13 to find a partition $\left\{\Lambda_{1}, \ldots, \Lambda_{s}\right\}$ of $\Lambda$ such that $\#\left(\Lambda_{1}\right)<\infty$, and for each $i$, either $\#\left(\Lambda_{i}\right)=\infty$ or $M \leq \#\left(\Lambda_{i}\right) \leq 2 q M^{2}$. Then

$$
\mathcal{P}(\Gamma) \cup\left\{\Lambda_{i}: M \leq \#\left(\Lambda_{i}\right) \leq 2 q M^{2}\right\}
$$

is an element in $\mathfrak{P}$ which is strictly larger than $\mathcal{P}(\Gamma)$ because $\#\left(\Lambda_{1}\right)<\infty$. Hence $\mathcal{P}(\Gamma)$ is not a maximal element, which is a contradiction. The theorem follows.

In the proof of Theorem 6.11 we only used the fact that $\Gamma$ is a connected graph of degree $q$. But each triangulation of a surface $S$ can be represented by a graph of degree 3 , so the following theorem may be proved by using the same method. 
Theorem 6.14. Suppose that $S$ is an open simply-connected Aleksandrov surface of curvature at most $k$. If $S$ has a tiling $\mathcal{T}$ satisfying conditions (R1) and (R2) in Theorem [1.5] and there exist constants $\epsilon>0$ and $M>0$ such that

$$
\sum_{\Delta \subseteq C_{\alpha}} K(\Delta) \leq-\epsilon
$$

for every connected cluster $C_{\alpha}$ with $\#\left(C_{\alpha}\right) \geq M$, then $S$ is hyperbolic.

\section{ACKNOWLEDGEMENT}

The author thanks A. Eremenko and D. Drasin for their helpful advice and encouragement.

\section{REFERENCES}

[1] L. Ahlfors, Conformal Invariants, McGraw-Hill, New York, 1973. MR0357743 (50:10211)

[2] A. D. Aleksandrov, Isoperimetric inequalities for curved surfaces, C. R. (Doklady) Acad. Sci. USSR (N.S.) 47 (1945), pp. 235-238. MR0013543 (7:167f)

[3] A. D. Aleksandrov, Über eine Verallgemeinerung der Riemannschen Geometrie, Schr. Forschungsinst. Math. 1 (1957), pp. 33-84. MR0087119 (19:304h)

[4] A. D. Aleksandrov and V. A. Zalgaller, Intrinsic Geometry of Surfaces, AMS Transl. Math. Monographs, Vol. 15, Providence, RI, 1967. MR0216434 (35:7267)

[5] I. Benjamini, S. Merenkov and O. Schramm, A negative answer to Nevanlinna's type question and a parabolic surface with a lot of negative curvature, Proc. Amer. Math. Soc. 132 (2004), no. 3, pp. 641-647 MR2019938 (2004k:30096)

[6] V. N. Berestovskij and I. G. Nikolaev, Multidimensional Generalized Riemannian Spaces, In: Geometry IV. Encyclopaedia of Mathematical Sciences (Yu. G. Reshetnyak ed.) Vol. 70, Springer, Berlin, 1993, pp. 165-243. MR,1263965

[7] M. Bonk, Singular Surfaces and Meromorphic Functions, Notices of AMS 49 (2002), no. 6, pp. 647-657. MR.1908328

[8] M. Bonk and A. Eremenko, Schlicht regions for entire and meromorphic functions, J. Analyse Math. 77 (1999), pp. 69-104. MR1753483 (2002c:30037)

[9] M. Bonk and A. Eremenko, Uniformly hyperbolic surfaces, Indiana Univ. Math. J. 49 (2000) no. 1, pp. 61-80. MF 1777037 (2001g:53120)

[10] M. Bonk and A. Eremenko, Covering properties of meromorphic functions, negative curvature and spherical geometry, Ann. of Math. (2) 152 (2000) no. 2, pp. 551-592. MR1804531 (2002a:30050)

[11] Yu. D. Burago and V. A. Zalgaller, Geometric Inequalities, Springer, Berlin, 1988. MR0936419 (89b:52020)

[12] W. Chauvenet, A Treatise on Plane and Spherical Trigonometry, J. B. Lippincott Co., Philadelphia, 1850

[13] M. Coornaert, T. Delzant and A. Papadopoulos, Géométrie et théorie des groupes, LNM, Vol. 1441, Springer, Berlin, 1990. MR1075994 (92f:57003)

[14] W. K. Hayman, Meromorphic Functions, Clarendon Press, Oxford, 1964. MR0164038 $(29: 1337)$

[15] A. Huber, Zum potentialtheoretischen Aspekt der Alexandrowshen Flächentheorie, Comment. Math. Helv. 34 (1960), pp. 99-126. MR0115147 (22:5949)

[16] R. Nevanlinna, Analytic Functions, Springer-Verlag, New York-Berlin, 1970. MR0279280 $(43: 5003)$

[17] B. Oh, Linear Isoperimetric Inequality, Gromov Hyperbolicity and Riemann Surfaces of class $F_{q}$, preprint.

[18] Yu. G. Reshetnyak, Two-Dimensional Manifolds of Bounded Curvature, In: Geometry IV. Encyclopaedia of Mathematical Sciences (Yu. G. Reshetnyak ed.) Vol. 70, Springer, Berlin, 1993, pp. 3-163. MR1263964

[19] S. Stoilov, Leçons sur les principes topologiques de la théorie des fonctions analytiques, Gauthier-Villars, Paris, 1956. 
[20] O. Teichmüller, Untersuchungen über konforme und quasikonforme Abbildung, Dtsch. Math. 3, 1938, no. 6, pp. 621-678.

[21] J. Väisälä, Lectures on n-dimensional quasiconformal mappings, LNM, Vol. 229, SpringerVerlag, Berlin-New York, 1971. MR0454009|(56:12260)

Department of Mathematics, Purdue University, West Lafayette, Indiana 47907

E-mail address: boh@math.purdue.edu

Current address: Department of Mathematics, University of Washington, Box 354350, Seattle, Washington 98195-4350

E-mail address: bgoh@math.washington.edu 